\title{
AN INDECOMPOSABLE PLANE CONTINUUM WHICH IS HOMEOMORPHIC TO EACH OF ITS NONDEGENERATE SUBCONTINUA $\left(^{1}\right)$
}

\author{
BY \\ EDWIN E. MOISE
}

In $1921 \mathrm{~S}$. Mazurkiewicz $\left({ }^{2}\right)$ raised the question whether every plane continuum which is homeomorphic to each of its nondegenerate subcontinua is a simple arc. In the present paper this question will be answered in the negative. It has been pointed out $\left({ }^{3}\right)$ by $R$. $L$. Wilder that if a continuum which is not an arc has this property, then it is its own prime part. G. T. Whyburn has shown(4) that no continuum with this property separates the plane.

The present paper describes a family of topologically equivalent compact, indecomposable plane continua with the property in question. These continua are very similar (if not, in fact, topologically equivalent) to a continuum described by B. Knaster $\left(^{5}\right)\left({ }^{6}\right)$.

At the time that he wrote the present paper, the author was a student of Professor R. L. Moore. It is a pleasure for him to acknowledge here his gratitude to Professor Moore for the experience of working with one of the few great teachers.

First some definitions will be given.

Definition 1. Let $C$ be a collection of mutually exclusive open sets $c_{1}, c_{2}, \cdots, c_{k}$, such that $c_{i}$ and $c_{j}$ have a boundary point in common if and only if $i$ and $j$ are identical or consecutive integers. The collection $C$ is said to be a chain, and the sets $c_{i}$ are said to be its links. If $P$ and $Q$ are points of $c_{1}$ and $c_{k}$ respectively, then $C$ is said to be a chain from $P$ to $Q$. Two links are said to be adjacent if they have a boundary point in common. The end-links

Presented to the Suciety, December 29, 1947; received by the editors May 13, 1947.

(1) Presented to the Faculty of the Graduate School of the University of Texas in partial fulfillment of the requirements for the degree of Doctor of Philosophy.

(2) Fund. Math. vol. 2 (1921) p. 285, Problem 14.

(3) R. L. Wilder, Some unsolved problems of topology, Amer. Math. Monthly vol. 44 (1937) p. 61.

(4) G. T. Whyburn, A continuum every subcontinuum of which separates the plane, Amer. J. Math. vol. 52 (1930) p. 319.

(5) B. Knaster, Un continu dont tout sous-continu esl indecomposable, Fund. Math. vol. $3^{\circ}$ (1922) p. 247.

( ${ }^{\circ}$ After this paper had been presented to a seminar at the University of 'Texas, and before it was submitted for publication, R. H. Bing observed that the continua described also settle the question whether every bounded homogeneous plane continuum is a simple closed curve. He is publishing this result elsewhere. 
of $C$ are $c_{1}$ and $c_{k}$. It is to be particularly noted that a link of a chain is not necessarily a connected set.

Definition 2. If $C$ and $D$ are finite collections of mutually exclusive open sets, and each element of $D$ is a subset of an element of $C$, then $D$ is said to be a refinement of $C\left({ }^{7}\right)$.

Definition 3. Let $C$ and $D$ be finite collections of mutually exclusive open sets, such that (1) each element of $D$ is the interior of the sum of the closures of one or more elements of $C$, and (2) $C^{*}$ is a subset of $D^{*}$. Then $D$ is said to be a consolidation of $C$. ( $C^{*}$ is the set of all elements of elements of

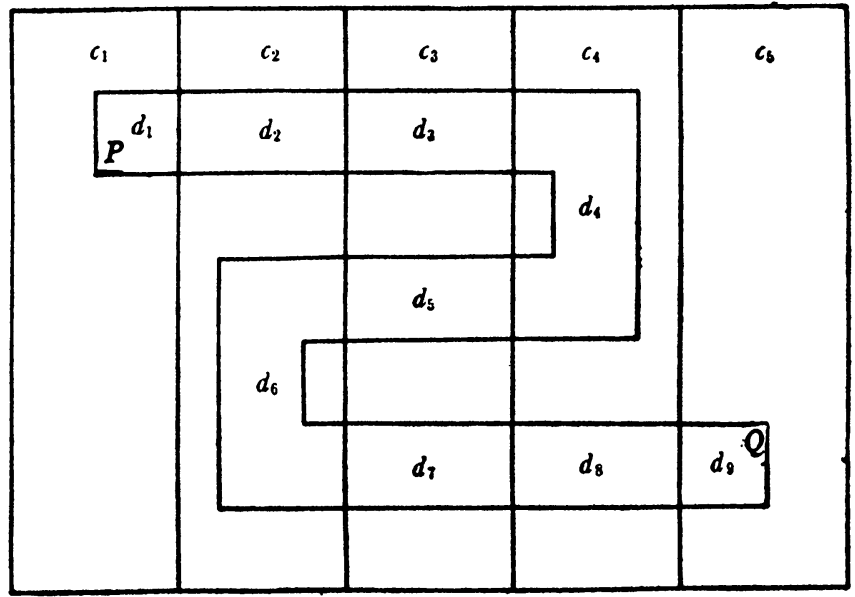

FIG. 1

This illustrates Definitions 6 and 7. The inner chain $D$ is very crooked with respect to the outer chain $C$. If $C$ is regarded as the chain $Y_{1}$ of Definition 7 , then the chain $Y_{2}$ may be obtained by splitting up each link of $D$ into five links by means of lines which do not intersect the boundaries between links of $D . D$ is thus the amalgam of $Y_{2}$ with respect to $Y_{1}$, in the sense of Definition 9.

$C$. If $C$ were a family of collections of sets, then $C^{* *}$ would be the set of all elements of elements of elements of $C\left({ }^{8}\right)$.)

Definition 4. Let $C, D$, and $E$ be finite collections of mutually exclusive open sets, such that $D$ is a refinement of $C$ and a consolidation of $E$. Then $D$ is said to be a proper consolidation of $E$ with respect to $C$.

Definition 5. Let the chain $D$ be a refinement of the chain $C$, such that for each link $c$ of $C$, the set of all links of $D$ that lie in $c$ is a subchain of $D$. Then $D$ is said to be straight with respect to $C$.

( ${ }^{7}$ This definition of a refinement is slightly different from various definitions given in the literature. Cf. L. Pontrjagin, Topological groups, p. 209, and S. Lefschetz, Algebraic topology, p. 13.

(8) This notation is due to R. L. Moore. See Foundations of point set theory, Amer. Math. Suc. Colloquiium Publications, vol. 13, p. 110. 
Definition 6. The following is a definition by induction: Let $C$ be a chain from $P$ to $Q$ whose links are $c_{1}, c_{2}, \cdots, c_{k}$. (1) If $C$ consists of less than five links, then a chain $D$ from $P$ to $Q$ is said to be very crooked with respect to $C$ if $D$ is straight with respect to $C$. (2) If $C$ is a chain from $P$ to $Q$ which consists of $k$ links, with $k$ greater than four, then a chain $D$ from $P$ to $Q$ is said to be very crooked with respect to $C$ if $D$ is a refinement of $C$, and $D$ is the sum of (i) a chain from $P$ to a point $x$ of $c_{k-1}$, (ii) a chain from $x$ to a point $y$ of $c_{2}$, and (iii) a chain from $y$ to $Q$, such that these chains are very crooked with respect to $C-c_{k}, C-c_{1}-c_{k}$, and $C-c_{1}$ respectively, and such that no two of them have in common any link that is not an end-link of both of them. (See Fig. 1.)

THEOREM 1. Let the chain $B$ be very crooked with respect to a chain $A$. Let $a$ and $a^{\prime}$ be links of $A$. Let $B^{\prime}$ be $a$ subchain of $B$ which is irreducible with respect to the property of being a chain from a point of a to a point of $a^{\prime}$. Then $B^{\prime}$ is very crooked with respect to the subchain $A^{\prime}$ of $A$ which has $a$ and $a^{\prime}$ as its end-links.

Proof. Let $A$ and $A^{\prime}$ consist of $n$ and $n^{\prime}$ links respectively. If $n^{\prime}$ is equal to $n$, the theorem follows by an easy induction on $n$. We shall complete the proof by an induction on $n-n^{\prime}$.

(1) If $A^{\prime}$ contains an end-link $a_{1}$ of $A$, then either $n^{\prime}$ is $n$ or $B^{\prime}$ is a subchain of the subchain $B_{1}$ of $B$ described in Definition 6, condition (2) (i). This case therefore reduces to the case $n^{\prime}, n-1$.

(2) If $A^{\prime}$ contains no end-link of $A$, then $B^{\prime}$ is a subchain of some one of the chains described in Definition 6, condition (2). This case therefore reduces to the case $n^{\prime}, n-1$.

Definition 7. Let $Y_{1}, Y_{2}, \cdots$ be a sequence of chains from $P$ to $Q$, such that (1) $\mathbb{S}\left(Y_{1}^{*}\right)$ is a compact metric space, (2) for each $i, Y_{i+1}$ is very crooked with respect to $Y_{i}$, and $\mathbb{S}\left(Y_{i+1}^{*}\right)$ lies in the interior of $\mathbb{S}\left(Y_{i}^{*}\right)$, (3) $Y_{1}$ consists of five links, (4) if $y$ is a link of $Y_{i}$, and $X$ is a subchain of $Y_{i+1}$ which is maximal with respect to the property of being a subchain of $Y_{i+1}$ and a refinement of the chain whose only link is $y$, then $X$ consists of five links, and (5) for each $i$, each link of $Y_{i}$ has diameter less than $1 / i$. Let $M$ be the common part of the sets $\mathbb{S}\left(Y_{i}^{*}\right)$. Then $M$ is said to be a pseudo-arc. (See Fig. 1.) (For reasons of typographical convenience, the closure of a set $M$ is denoted by $\mathbb{(}(M)$.)

Definition 8. Let $G$ and $G^{\prime}$ be finite collections of mutually exclusive open sets, such that $G^{\prime}$ is a refinement of $G$. Let $H$ and $H^{\prime}$ have the same properties. Let $T$ and $T^{\prime}$ be reversible transformations such that (1) $T(G)$ is $H$, and $T^{\prime}\left(G^{\prime}\right)$ is $H^{\prime},(2)$ two elements $x$ and $y$ of $G$ (or $G^{\prime}$ ) have a boundary point in common if and only if $T(x)$ and $T(y)$ (or $T^{\prime}(x)$ and $T^{\prime}(y)$ ) have a boundary point in common, and (3) an element $g$ of $G$ contains an element $g^{\prime}$ of $G^{\prime}$ if and only if $T(g)$ contains $T^{\prime}\left(g^{\prime}\right)$. Then $G^{\prime}$ and $H^{\prime}$ are said to be 
similar with respect to $G$ and $H$ under the transformations $T$ and $T^{\prime}$.

THEOREM 2. Let $G_{1}, G_{2}, \cdots$ and $H_{1}, H_{2}, \cdots$ be sequences of finite collections of mutually exclusive open sets in compact metric spaces $G$ and $H$ respectively, and let $T_{1}, T_{2}, \cdots$ be a sequence of reversible transformdtions, such that (1) for each $i, \mathfrak{S}\left(G_{i}^{*}\right)$ is $G$ and $\mathbb{S}\left(H_{i}^{*}\right)$ is $H,(2)$ there is a sequence $f_{1}, f_{2}, \ldots$ of positive numbers which converges to 0 , such that each element of $G_{i}+H_{i}$ has diameter less than $f_{i},(3) G_{i+1}$ and $H_{i+1}$ are refinements of $G_{i}$ and $H_{i}$ respectively, and (4) $G_{i+1}$ and $H_{i+1}$ are similar with respect to $G_{i}$ and $H_{i}$ under the transformations $T_{i}$ and $T_{i+1}$. Then $G$ and $H$ are topologically equivalent. It should be noted that the elements of $G_{i}$ and $H_{i}$ are not necessarily connected.

Proof. Let $P$ be a point of $G$. Let $D_{1}$ be the interior of $\mathfrak{C}\left(E_{1}^{*}\right)$, where $E_{1}$ is the set of all elements $g$ of $G_{1}$ such that $P$ belongs to $\mathbb{C}(g)$. Then $P$ belongs to $D_{1}$. Given $D_{i}$, let $j$ be an integer greater than $i$, such that if $g$ belongs to $G_{j}$ and $P$ belongs to $\mathbb{S}(g)$, then $\mathbb{S}(g)$ is a subset of $D_{i}$. Let $D_{i+1}$ be the interior of $\mathbb{E}\left(E_{j}^{*}\right)$, where $E_{j}$ is the set of all elements $g$ of $G_{j}$ such that $P$ belongs to $\mathfrak{S}(g)$. Then $\mathbb{E}\left(D_{i+1}\right)$ is a subset of $D_{i}$. Since each element of $E_{j}$ has a boundary point in common with each other element of $E_{j}, D_{i}$ has diameter less than $3 f_{i}$. Therefore the sequence $D_{1}, D_{2}, \cdots$ closes down on $P$.

Now for each $i$, let $D_{i}^{\prime}$ be the interior of $\mathbb{E}\left(T_{j}\left(E_{j}\right)^{*}\right)$. If $D_{i+1}$ is the interior of $\mathfrak{S}\left(E_{k}^{*}\right)$, and $g^{\prime}$ belongs to $E_{k}$, then there is a $g$ of $E_{j}$ such that $g$ contains $g^{\prime}$. It can be shown (by an easy induction on $k-j$ ) that $G_{k}^{\prime}$ and $H_{k}^{\prime}$ are similar with respect to $G_{j}$ and $H_{j}$ under the transformations $T_{j}$ and $T_{k}$. It follows that $T_{j}(g)$ contains $T_{k}\left(g^{\prime}\right)$, so that the $D^{\prime}$-sequence is monotonic. If $h$ belongs to $G_{j}-E_{j}$, and $h^{\prime}$ belongs to $G_{k}$ and is a subset of $h$, and $g^{\prime}$ belongs to $E_{k}$, then $g^{\prime}$ and $h^{\prime}$ have no boundary point in common. Since $H_{k}$ is finite, it follows that $\mathbb{E}\left(D_{i+1}^{\prime}\right)$ is a subset of $D_{i}^{\prime}$. Since each two elements of $E_{j}$ have a boundary point in common, it follows that each two elements of $T_{j}\left(E_{j}\right)$ have a boundary point in common, so that the diameter of $D_{i}^{\prime}$ is less than $3 f_{i}$. Therefore the sequence $D_{1}^{\prime}, D_{2}^{\prime}, \cdots$ closes down on a point. Let this point be $U(P)$.

We shall show that $U$ is a one-to-one correspondence. Let $P$ and $P^{\prime}$ be points of $G$. Let $i$ be such that if $g$ and $g^{\prime}$ are elements of $G_{i}$, and $P$ belongs to $\mathbb{E}(g)$, and $P^{\prime}$ belongs to $\mathbb{E}\left(g^{\prime}\right)$, then $\mathbb{E}(g)$ and $\mathbb{E}\left(g^{\prime}\right)$ have no point in common. It follows that $\mathbb{E}\left(T_{i}(g)\right)$ and $\mathbb{E}\left(T_{i}\left(g^{\prime}\right)\right)$ have no point in common, so that $U(P)$ is not $U\left(P^{\prime}\right)$.

We now wish to show that the transformation $U$ is continuous. Let the point $P$ of $G$ be the sequential limit point of the sequence $P_{1}, P_{2}, \ldots$. Let $R$ be an open set in $H$ which contains $U(P)$. Let $i$ be such that $R$ contains the sphere of radius $3 f_{i}$ whose center is $U(P)$. Let $E_{i}$ be the set of all elements $g$ of $G_{i}$ for which $P$ belongs to $\left(\mathfrak{C}(g)\right.$, and let $Z$ be the interior of $\mathfrak{S}\left(E_{i}^{*}\right)$. Then there is an $n$ such that $P_{n}$ belongs to $Z$. It follows that $U\left(P_{n}\right)$ belongs to $R$. Therefore $U$ is a continuous transformation. 
It is a known theorem that if $S$ and $S^{\prime}$ are compact metric spaces, and $V$ is a one-to-one continuous transformation such that $V(S)$ is $S^{\prime}$, then $V$ is a homeomorphism. Therefore $G$ and $H$ are topologically equivalent.

Definition 9. Let $C$ be a chain, and let $D$ be a chain which is a refinement of $C$. Then the amalgam of $D$ with respect to $C$ is the set of all interiors of sets $c \cdot\left(d^{*}\right)$, where $c$ is a link of $C$ and $d$ is a subchain of $D$ which is maximal with respect to the property of being a subchain of $D$ and a refinement of the chain whose only link is $c$.

TheOREM 3. Let $C, D, E$, and $F$ be chains, such that (1) $C$ and $E$ consist of the same number of links, and (2) $D$ is very crooked with respect to $C$, and $F$ is very crooked with respect to $E$. Let $D^{\prime}$ be the amalgam of $D$ with respect to $C$, and let $F^{\prime}$ be the amalgam of $F$ with respect to $E$. Let $T$ be a reversible transformation preserving adjacency, such that $T(C)$ is $E$. Then there is a transformation $T^{\prime}$ such that $D^{\prime}$ and $F^{\prime}$ are similar with respect to $C$ and $E$ under the transformations $T$ and $T^{\prime}$.

Indication of proof. Let $C$ consist of $n$ links. We show by induction on $n$ that $D^{\prime}$ and $F^{\prime}$ are very crooked with respect to $C$ and $E$ respectively. The proof may be completed by another induction on $n$.

Theorem 4. Let $M$ and $N$ be pseudo-arcs, and let $C_{1}, C_{2}, \cdots$ and $D_{1}, D_{2}, \cdots$ be sequences satisfying Definition 7 with respect to $M$ and $N$ respectively. Then there is a sequence $T_{1}, T_{2}, \cdots$ of transformations such that for each $i, C_{i+1}$ and $D_{i+1}$ are similar with respect to $C_{i}$ and $D_{i}$ under the transformations $T_{i}$ and $T_{i+1}$.

This may be proved by means of Theorem 3 and condition (4) of Definition 7.

TheOREM 5. If $M$ and $N$ are pseudo-arcs, then $M$ and $N$ are topologically equivalent.

Theorem 5 may be proved with the help of Theorems 2 and 4 , the point sets $M$ and $N$ being regarded as spaces.

THEOREM 6. Every pseudo-arc is a compact continuum which contains no decomposable continuum.

Proof. It is clear that a pseudo-arc $M$ is closed and compact. Suppose that $M$ is the sum of two mutually exclusive closed point-sets $H$ and $K$. There is a positive integer $i$ such that $3 / i$ is less than the shortest distance from a point of $H$ to a point of $K$. Clearly $Y_{i}$ is not a chain. This involves a contradiction.

The rest of the proof will be indicated briefly. Let $N$ be a subcontinuum of $M$, and for each $i$ let $Y_{i}^{\prime}$ be the chain which consists of all links of $Y_{i}$ that contain a point of $N$. Let $K$ be a proper subcontinuum of $N$, and for each $i$ let 
$Y_{i}^{\prime \prime}$ be the subchain of $Y_{i}^{\prime}$ which consists of all links of $Y_{i}$ that contain a point of $K$. For all but a finite number of integers $i, Y_{i}^{\prime}-Y_{i}^{\prime \prime}$ contains two adjacent links of $Y_{i}^{\prime}$. For such an $i$, the set of all links of $Y_{i+1}^{\prime}$ which lie in links of $Y_{i}^{\prime \prime}$ contains two chains which "lie close together," such that one of them has $Y_{i+1}^{\prime \prime}$ as a refinement. From this it is not hard to show that $K$ is a continuum of condensation of $N$; that is to say, $\mathbb{}(N-K)$ is $N$. Therefore $N$ is indecomposable $\left({ }^{9}\right)$.

\section{THEOREM 7. There is a pseudo-arc in the plane.}

Definition 10. If $M$ is a pseudo-arc, and $Y_{1}, Y_{2}, \cdots$ is a sequence of chains satisfying Definition 7 , then $C_{1}, C_{2}, \cdots$ denotes a sequence of collections of sets such that $c$ belongs to $C_{i}$ if and only if $c$ is the common part of $M$ and a link of $Y_{i}$. If $M$ is regarded as space, it is clear that for each $i$, $C_{i}$ is a chain, and that $C_{i+1}$ is a proper consolidation of $C_{i+2}$ with respect to $C_{i}$. Moreover, there is a sequence $T_{1}, T_{2}, \cdots$ of reversible transformations, such that $C_{i+1}$ and $Y_{i+1}$ are similar with respect to $C_{i}$ and $Y_{i}$ under the transformations $T_{i}$ and $T_{i+1}$. Throughout the following discussion, $M$ will be regarded as space.

In the ensuing arguments, we wish to show that given a subcontinuum $N$ of $M$, we can represent $M$ and $N$ by means of sequences of chains, these sequences being structurally similar in such a sense as to satisfy the hypothesis of Theorem 2. The first step will be to show that there are points $R$ and $S$ of $N$ which "lie at opposite ends of $N$." It is not obvious that this is true. If for each $i$ we let $C_{i}^{\prime}$ be the set of all links of $C_{i}$ that contain a point of $N$, then we can define a subcontinuum of $M$ whose sequence $C_{1}^{\prime}, C_{2}^{\prime}, \cdots$ is structurally very different from the sequence $C_{1}, C_{2}, \ldots$; for example, it is possible that no end-link of any chain $C_{i}^{\prime}$ contains an end-link of any other chain of the sequence. This difficulty is avoided by means of the following theorem. The points $R$ and $S$ will turn out to be the images of $P$ and $Q$ under a homeomorphism which maps $M$ on $N$.

THEOREM 8. Let the continuum $M$ and the sequence $C_{1}, C_{2}, \cdots$ satisfy Definition 10. Let $N$ be a subcontinuum of $M$. Then there are points $R$ and $S$ of $N$, and a sequence $D_{1}, D_{2}, \cdots$ of chains from $R$ to $S$, such that (1) the common part of the sets $\mathbb{S}\left(D_{i}^{*}\right)$ is $N$, (2) for each $i, D_{i+1}$ is a refinement of $D_{i}$ and also a refinement of $C_{i}$, (3) for each $i$, there is a $j$ such that $D_{i}$ is a consolidation of a subset of $C_{j}$, and (4) for each $i, R$ and $S$ belong to $C_{i}^{*}$.

Proof. For each $i$, let $C_{i}^{\prime}$ be the subchain of $C_{i}$ which consists of all links of $C_{i}$ that contain a point of $N$. Let $j$ be an integer such that $C_{j}^{\prime}$ consists of at least three links; and let $D_{1}$ be $C_{j}^{\prime}$. Let $d_{1}$ and $d_{1}^{\prime}$ be end-links of $D_{1}$. Sup-

(9) S. Janiszewski and C. Kuratowski, Sur les continus indécomposables, Fund. Math. vol. 1 (1920) p. 212, Theorem II. The latter part of the proof of Theorem 6 is very similar to a proof given by Knaster, loc. cit. p. 279. 
pose that we have given $D_{m}, d_{m}$, and $d_{m}^{\prime}(m=1,2, \cdots, i)$, such that (1) $d_{m}$ and $d_{m}^{\prime}$ are the end-links of $D_{m},(2) \quad\left(S\left(d_{m+1}\right)\right.$ is a subset of $\left(d_{m}\right)$, and (3) $\mathfrak{E}\left(d_{m+1}^{\prime}\right)$ is a subset of $d_{m}^{\prime}$. Let $j$ be an integer greater than $i$, such that $C_{j}^{\prime}$ is a refinement of $D_{i}$, and let the links of $C_{j}^{\prime}$ be $c_{1}, c_{2}, \cdots, c_{q}$. Let $c_{r}$ be the first link of $C_{j}^{\prime}$ which is a subset of an end-link of $D_{i}$, and suppose, without loss of generality, that $c_{r}$ is a subset of $d_{i}$. There is an integer $u$ such that $C_{u}^{\prime}$ contains a subchain $K$ which has the following properties: (1) $\mathfrak{E}\left(K^{*}\right)$ lies in $\subseteq\left(c_{1}+c_{2}+\cdots+c_{r}\right),(2)$ the end-links of $K$ lie in $c_{1}$, and (3) there is a link $k$ of $K$ such that $\mathbb{E}(k)$ lies in $\left.d_{n}{ }^{10}\right)$. Moreover, any integer greater than $u$ will satisfy the same conditions. (See Fig. $2\left({ }^{11}\right)$ ).

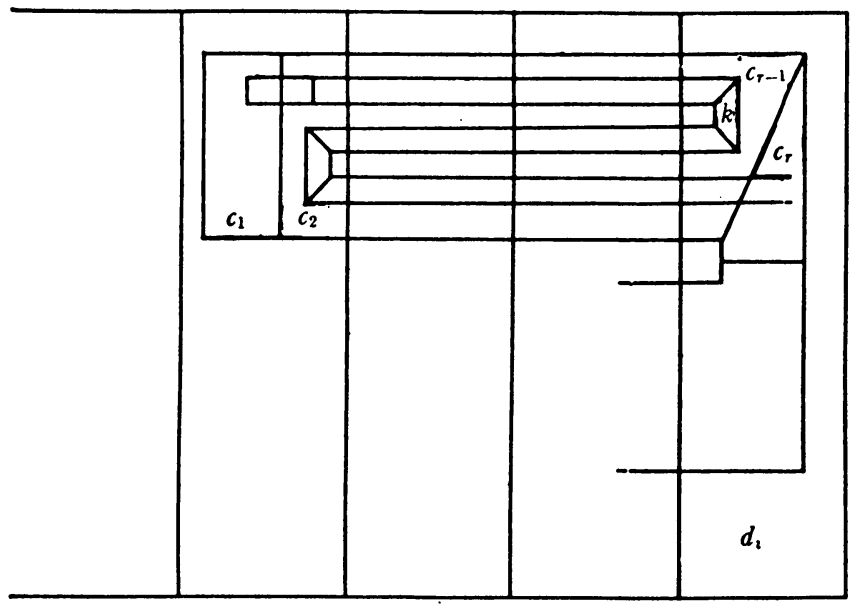

FIG. 2

This illustrates part of the procedure used in the proof of Theorem 8, to obtain $D_{i+1}$ from $D_{i}$. In order of diminishing size of links, the chains shown are $D_{i}, C_{j}^{\prime}$, and $C_{*}^{\prime}$.

Now let $s$ be the greatest integer $t$ for which $c_{t}$ is a subset of $d_{i}^{\prime}$. Let $v$ be an integer such that $C_{v}^{\prime}$ contains a chain $H$ such that (1) $\mathbb{E}\left(H^{*}\right)$ lies in $\mathfrak{S}\left(c_{s}+c_{s+1}+\cdots+c_{q}\right),(2)$ the end-links of $H$ lie in $c_{q}$, and (3) there is a link $h$ of $H$ such that $\mathbb{C}(h)$ lies in $d_{i}^{\prime}$. Let $w$ be the greater of the integers $u$ and $v$. Let $K^{\prime}$ and $H^{\prime}$ be subchains of $C_{w}^{\prime}$ having the properties required for $K$ and $H$, and let $k^{\prime}$ and $h^{\prime}$ be the links of $K^{\prime}$ and $H^{\prime}$ respectively satisfying the conditions (3). Let $d_{i+1}$ be $k^{\prime}$, and let $d_{i+1}^{\prime}$ be $h^{\prime}$. Let the other links of $D_{i+1}$ be the interiors of (1) $c_{1} \cdot \mathbb{E}\left(C_{w}{ }^{*}\right),(2) c_{q} \cdot \mathfrak{S}\left(C_{w}{ }^{*}\right)$, (3) the sets $c_{n} \cdot \mathbb{S}\left(K^{\prime *}-k^{\prime}\right)$ and $c_{n} \cdot \mathbb{E}\left(H^{\prime *}-h^{\prime}\right)$, except for such of these sets as may be empty, and (4) the sets $c_{n} \cdot \mathfrak{C}\left(C_{j}^{*}-K^{*}-H^{\prime *}\right)$, where $n$ is different from 1 and from $q$.

(10) See Theorem 1. Note that $C^{\prime}{ }_{j+1}$ does not necessarily have these properties.

(11) This and all following figures have been simplified in so many ways as to be hardly more than graphic memoranda of the notation used in the text. 
The sequence $D_{1}, D_{2}, \cdots$ is now defined. For each $i, D_{i}$ is a consolidation of a certain $C_{j}^{\prime}$, namely, the $C_{w}^{\prime}$ used in the transitivity proof above. $R$ and $S$ are the common parts of the sets $d_{i}$ and the sets $d_{i}^{\prime}$ respectively.

Of course the $D$-sequence representing $N$ is not in general structurally similar to the $C$-sequence representing $M$ : corresponding chains may not consist of the same number of links; and the chains of the $D$-sequence may fail to be very crooked in the sense of Definition 6, possibly by being "too crooked." The first and lesser of these difficulties is met by Theorem 11, and the second by Theorem 9. The generality and complication of Theorem 9 were introduced in order to permit an induction proof; the corollary is what will actually be used.

THEOREM 9. Hypothesis: Let $D$ be a chain from $R$ to $S$, and let $D^{\prime}$ be a chain from $R$ to $S$ which is a refinement of $D$, such that if $d^{\prime}$ and $d^{\prime \prime}$ are links of $D^{\prime}$ which lie in the same link of $D$, then $d^{\prime}$ and $d^{\prime \prime}$ are not adjacent. Let $E$ be a chain which consists of the same number of links as $D$, such that $E$ is a consolidation of a subset of a certain $C_{j}$, and let $T$ and $U$ be points belonging to different endlinks $e_{1}$ and $e_{m}$ of $E$. Let $A$ be a reversible transformation such that $A(D)$ is $E$, and such that two links of $D$ are adjacent if and only if their images under $A$ are adjacent. For each $i$ greater than $j$, let $G_{i}$ be a collection of mutually exclusive subchains of $C_{i}$, such that (1) if $g$ and $g^{\prime}$ are links of different chains of $G_{i}$, then $g$ and $g^{\prime}$ are not adjacent, (2) $G_{i}^{*}$ is a refinement of $E$, (3) $G_{i}$ contains a chain two of whose links contain $T$ and $U$, (4) $G_{i+1}^{* *}$ is a subset of $G_{i}^{* *}$, (5) if $g$ is a subchain of a chain of $G_{i}$, then there is a subchain $g^{\prime}$ of a chain of $G_{i+1}$ which has an end-link in each end-link of $g$, such that $g^{\prime}$ is a refinement of $g$, and (6) if $K$ is a chain from $T$ to a point not in $e_{1}$, or from $U$ to a point not in $e_{m}$, and $K$ is a subchain of a chain of $G_{j+1}$, then $K$ consists of at least four links.

Conclusion: There is a chain $E^{\prime}$ from $T$ to $U$, a iransformation $A^{\prime}$, and an integer p, such that (1) $E^{\prime}$ is a refinement of $E$, (2) $G_{p}^{*}$ is a refinement of $E^{\prime}$, (3) the end-links of $E^{\prime}$ contain the elements of $G_{j+1}^{*}$ that contain $T$ and $U$, and (4) $D^{\prime}$ and $E^{\prime}$ are similar with respect to $D$ and $E$ under the transformations $A$ and $A^{\prime}$.

In the proof of this theorem we shall use the following lemma:

Lemma. Let $g$ be a subchain of a chain $G$ of $G_{i}$, such that neither end-link of $G$ belongs to $g$. Let $E_{g}$ be the subchain of $E$ which consists of all links of $E$ that contain a link of $g$. Let $\gamma$ and $\gamma^{\prime}$ be links of $g$ which lie in different end-links $z$ and $z^{\prime}$ of $E_{g}$, such that each subchain of $g$ from a point of $\gamma$ to a point not in $z$, or from a point of $\gamma^{\prime}$ to a point not in $z^{\prime}$, consists of at least four links. For each $k$ greater than $i$, let $G_{k}{ }^{\prime}$ be the set of all subchains of chains of $G_{i}$ which are maximal with respect to the property of being refinements of $g$. Then there are points $T^{\prime}$. and $U^{\prime}$ such that $E_{g}, T^{\prime}, U^{\prime}$, and the sequence $G_{i+1}^{\prime}, G_{i+2}^{\prime}, \cdots$ satisfy all the conditions required for $E, T, U$, and the sequence $G_{j+1}, G_{i+2}^{\prime}, \cdots$. 
Proof of lemma. First we note that since $E$ is a consolidation of a subset of $C_{j}, E_{g}$ is a consolidation of a subset of $C_{i-1}$. Since neither end-link of $G$ belongs to $g$, condition (5) of the hypothesis gives us a subcontinuum $N^{\prime}$ of $M$ which lies in $\mathbb{S}\left(g^{*}\right)$ and contains a point $T^{\prime}$ of $\gamma$ and a point $U^{\prime}$ of $\gamma^{\prime}$, such that $T^{\prime}$ and $U^{\prime}$ satisfy condition (3) with respect to the collections $G_{k}^{\prime}$. The other conditions are easily verified.

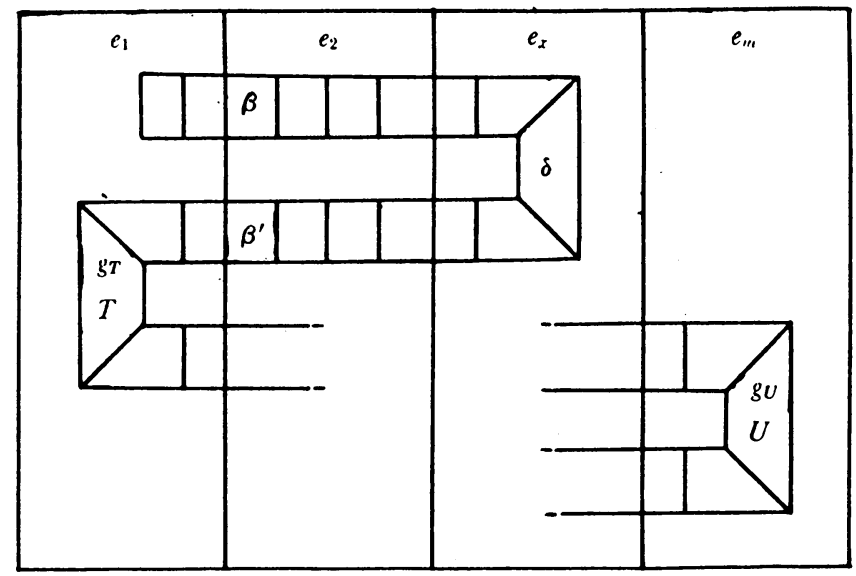

FIG. 3

This illustrates a part of the proof of Theorem 9 in case (I). The chain $h$ has $\beta$ and $\beta^{\prime}$ as its end-links.

Proof of theorem. We shall prove the theorem by an induction on $m+n$, where $m$ is the number of links of $D$ and $n$ is the number of links, of $D^{\prime}$. If $m+n$ is 2 , the theorem is self-evident. For the transitivity proof, we shall assume that $D$ has $m$ links and $D^{\prime}$ has $n$ links, and assume that the theorem is true for any $m^{\prime}, n^{\prime}$ for which $m^{\prime}+n^{\prime}$ is less than $m+n$. The proof now falls in to two parts.

(I) Suppose that there is an end-link of $D$ which contains only one link of $D^{\prime}$. Without loss of generality, suppose that this is the link $d_{1}$ of $D$ that contains $R$, and that $A\left(d_{1}\right)$ is $e_{1}$, where $e_{1}$ contains $T$. Let $d_{1}^{\prime}$ be the link of $D^{\prime}$ that contains $R$. Let the link $e_{1}^{\prime}$ of $E^{\prime}$ that contains $T$ be the interior of $e_{1} \cdot \mathfrak{c}\left(G_{j+1}^{* *}\right)$.

Let $H$ be the set of all subchains $h$ of chains of $G_{j+1}$ which are maximal with respect to the property of being refinements of $E-e_{1}$. The chains $h$ are of three types.

(1) If two links $\beta$ and $\beta^{\prime}$ of $h$ have boundary points in common with elements of $G_{j+1}^{*}$ which lie in $e_{1}$, and $e_{x}$ is the last link of $E$ that contains a link of $h$, then there is a link $\delta$ of $h$ which lies in $e_{x}$, such that any subchain of $h$ 
from a point of $\delta$ to a point not in $e_{x}$ consists of at least four links( $\left.{ }^{12}\right)$. Therefore $h$ is the sum of a chain $h^{\prime}$ which has $\beta$ and $\delta$ as its end-links and a chain $h^{\prime \prime}$ which has $\beta^{\prime}$ and $\delta$ as its end-links; and each of these chains satisfies the hypothesis of the preceding lemma. (See Fig. 3.) Let $D_{h}^{\prime}$ be the subchain of $D^{\prime}$ which contains $d_{2}^{\prime}$ and is maximal with respect to the property of being a refinement of the subchain $D_{h}$ of $D$ which has $d_{2}$ and $d_{x}$ as its end-links. For each $i$ greater than $j+1$, let $G_{i}^{\prime}$ and $G_{i}^{\prime \prime}$ be the sets of all subchains of chains of $G_{i}$ which are maximal with respect to the property of being refinements of $h^{\prime}$ and $h^{\prime \prime}$ respectively. By virtue of the lemma and the induction hypothesis, there is a chain $E_{h^{\prime}}^{\prime}$, and an integer $v$, such that (1) $E_{h^{\prime}}^{\prime}$ is a refinement of $E$, (2) $G_{v}^{\prime *}$ is a refinement of $E_{h^{\prime}}^{\prime}$, (3) the end-links of $E_{h^{\prime}}^{\prime}$ contain $\beta$ and $\delta$, and (4) $D_{h}^{\prime}$ and $E_{h^{\prime}}^{\prime}$ are similar with respect to $D$ and $E$ under a pair of transformations one of which is $A$.

For the same reasons, there is a chain $E_{h^{\prime \prime}}^{\prime}$, and an integer $w$, such that $E_{h^{\prime \prime}}^{\prime \prime}$ satisfies analogous conditions with respect to $G_{w}{ }^{\prime \prime}$.

The two chains thus obtained are similar with respect to $E$ and $E$ under a pair of transformations one of which is the identity for each link of $E$. We add together the links which correspond under the similarity transformation, thus obtaining a chain $E_{h}^{\prime}$ which is similar to $D_{h}^{\prime}$ with respect to $E$ and $D$ under a pair of transformations one of which is $A$. (Since $h$ was a chain to begin with, no new adjacency relations are introduced by the addition.)

Now $D_{h}^{\prime}+d_{1}^{\prime}$ and $E_{h}^{\prime}+e_{1}^{\prime}$ are similar with respect to $D$ and $E$ under a pair of transformations one of which is $A$.

(2) If only one link $\beta$ of $h$ has a boundary point in common with an element of $G_{j+1}^{*}$ which lies in $e_{1}$, let $e_{x}$ be the last link of $E$ that contains a link of $h$. If there is a link $\delta$ of $h$ which lies in $e_{x}$, such that any subchain of $h$ from a point of $\delta$ to a point of $e_{x-1}$ consists of at least four links, we proceed as with $h^{\prime}$ above, using a trivial extension of the lemma. Otherwise, the set of all links of $h$ that lie in $e_{x}$ is a subchain of $h$ only one of whose links has a boundary point in common with $e_{x-1}$. Let $\delta$ be the last link of $h$ that does not lie in $e_{x}$, and let $h^{\prime}$ be the subchain of $h$ whose end-links are $\beta$ and $\delta$. Let $D_{h}^{\prime}$ be the. subchain of $D^{\prime}$ which is irreducible with respect to the property of containing $d_{2}^{\prime}$ and a link which lies in $d_{x}$, and let $D_{h^{\prime}}^{\prime}$ be $D_{h}^{\prime}-z$, where $z$ is the last link of $D_{h}^{\prime}$. Proceed as before to obtain a chain $E_{h^{\prime}}^{\prime}$ and an integer $w$ such that (1) $E_{h^{\prime}}^{\prime}$ is a refinement of $E$, (2) each element of $G_{w}{ }^{*}$ that lies in a link of $h^{\prime}$ is a subset of a link of $E_{h^{\prime}}^{\prime}$, (3) the end-links of $E_{h^{\prime}}^{\prime}$ contain $\beta$ and $\delta$, and (4) $D_{h^{\prime}}^{\prime}$ and $E_{h^{\prime}}^{\prime}$ are similar with respect to $D$ and $E$ under a pair of transformations one of which is $A$.

Now $D_{h^{\prime}}^{\prime}+d_{1}^{\prime}+z$ and $E_{h^{\prime}}^{\prime}+e_{1}^{\prime}+e_{x} \cdot Y$ (where $Y$ is the interior of $\subseteq\left(h^{*}\right)$ ) are similar with respect to $D$ and $E$ under a pair of transformations one of which is $A$.

(3) The remaining chains of $H$ may be taken care of all at once by con-

(12) See Definition 7, condition (4). 
sidering them together with the chain $h$ of $H$ for which $U$ belongs to $h^{*}$; we use an easy extension of the lemma.

Given any two chains obtained under (1), (2), or (3) above, one is similar to a subchain of the other with respect to $E$ and $E$ under a pair of transformations one of which is the identity for each link of $E$. We obtain the chain $E^{\prime}$ by adding together the links which correspond to one another under these transformations, and we let $p$ be the greatest integer employed as a subscript in the construction.

(II) Suppose tnat each end-link of $D$ contains at least two links of $D^{\prime}$. Then there is an integer $x$ such that $D^{\prime}$ is the sum of a chain $D_{1}^{\prime}$ from $R$ to a point $P_{x}$ of $d_{x}$, a chain $D_{2}^{\prime}$ from $P_{x}$ to a point $P_{1}$ of $d_{1}$, and a chain $D_{3}^{\prime}$ from $P_{1}$ to $S$, such that the first two of these chains are refinements of the subchain $D_{1, x}$ of $D$ which has $d_{1}$ and $d_{x}$ as its end-links.

Let $g$ be a subchain of a chain of $G_{j+1}$, such that $g$ contains $T$ and $U$ in its end-links $g_{T}$ and $g_{U}$. Let $g^{\prime}$ be the subchain of $g$ which contains $g_{T}$ and is maximal with respect to the property of being a refinement of the subchain $E_{1, x}$ of $E$ which has $e_{1}$ and $e_{x}$ as its end-links. Let the other end-link of $g^{\prime}$ be $z$. (Note that if $x$ is $m$, then $z$ is $g_{U}$.) Let $\gamma$ and $\gamma^{\prime}$ be the links of $g^{\prime}$ that are adjacent to $g_{T}$ and $z$ respectively. Then if $h$ is a subchain of a chain of $G_{j+2}$ which is irreducible with respect to the property of being a chain from a point of $g_{T}$ to a point of $z$, it follows that there are links $\delta$ and $\delta^{\prime}$ of $h$ such that (1) $\delta$ lies in $\gamma$, and $\delta^{\prime}$ lies in $\gamma^{\prime}$, (2) any subchain of $h$ which contains $\delta$ and a link not in $\gamma$, or $\delta^{\prime}$ and a link not in $\gamma^{\prime}$, consists of at least four links, and (3) $h$ is the sum of a refinement of $g^{\prime}-z$ which has $\delta^{\prime}$ and a subset of $g_{T}$ as its end-links, a refinement of $g^{\prime}-z-g_{T}$ which has $\delta$ and $\delta^{\prime}$ as its end-links, and a refinement of $g^{\prime}-g_{T}$ which has $\delta$ and a subset of $z$ as its end-links $\left({ }^{13}\right)$.

If $K$ is a subchain of a chain of $G_{j+2}$ which is irreducible with respect to the property of being a chain from a point of $g_{T}$ to a point of $g_{U}$, then $K$ contains a subchain $h$ which has the properties given above. Let the end-links of $K$ be $\beta$ and $\beta^{\prime}$. Then there are links $\delta$ and $\delta^{\prime}$ of $K$, lying in $e_{x}$ and $e_{1}$ respectively, such that any subchain of $K$ which contains $\delta$ and a link not in $\dot{e}_{x}$ (or $\delta^{\prime}$ and a link not in $e_{1}$ ) consists of at least four links. Moreover, $K$ is the sum of three chains $K_{1}, K_{2}$, and $K_{3}$, such that (1) the end-links of $K_{1}$ are $\beta$ and $\delta$, (2) the end-links of $K_{2}$ are $\delta$ and $\delta^{\prime}$, (3) the end-links of $K_{3}$ are $\delta^{\prime}$ and $\beta^{\prime}$, and (4) $K_{1}$ and $K_{2}$ are refinements of the subchain $E_{1, x}$ of $E$ whose end-links are $e_{1}$ and $e_{x}$. (See Fig. 4.)

If $y$ is 1,2 , or 3 , and $i$ is greater than $j+2$, let $G_{y, i}$ be the set of all subchains of chains of $G_{i}$ that are maximal with respect to the property of being refinements of $K_{y}$. As in case (I), there is an integer $w$, and chains $E_{K, y}^{\prime}$ such that (1) $E_{K, y}^{\prime}$ is a refinement of $E$, (2) $G_{y, w}^{*}$ is a refinement of $E_{K, y}^{\prime},(3)$ the endlinks of $E_{K, y}^{\prime}$ contain the end-links of $K_{y}$, and (4) $D_{y}^{\prime}$ and $E_{K, y}^{\prime}$ are similar with respect to $D$ and $E$ under a pair of transformations one of which is $A$. By adding together the intersecting end-links of these chains, we obtain a

(13) See definition 7(2), and Theorem 1. 
chain $E_{K}^{\prime}$ such that (1) $E_{K}^{\prime}$ is a refinement of $E$, (2) $G_{1, w}^{*}+G_{2, w}^{*}+G_{3, v}^{*}$ is a refinement of $E_{K}^{\prime}$, (3) the end-links of $E_{K}^{\prime}$ contain $g_{T}$ and $g_{U}$, and (4) $D^{\prime}$ and $E_{\mathbf{k}}^{\prime}$ are similar with respect to $D$ and $E$ under a pair of transformations one of which is $A$.

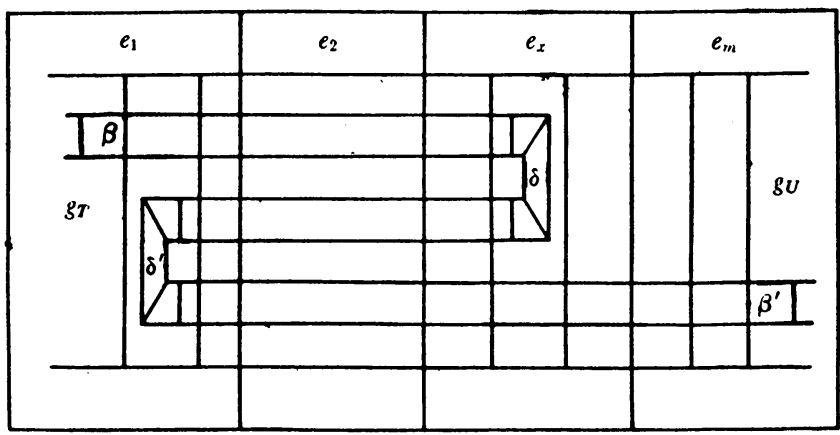

FIG. 4

This illustrates a part of the proof of Theorem 9 in case (II). In order of diminishing size of links, the chains shown are $E, g$, and a subchain of a chain of $G_{i+2}$. Note that the chain $g$ is pictured as being straight with respect to $E$; this would not generally be true.

Let $H$ be the set of all subchains $h$ of chains of $G_{j+2}$ which are maximal with respect to the property of containing no link which lies in $g_{T}$ or $g_{U}$ or belongs to a chain of type $K$ treated above. By means of the lemma, the induction hypothesis, and the hypothesis for $D^{\prime}$ in case (II), each chain $h$ of $H$ can be made to yield a chain $E_{h}{ }^{\prime}$ such that (1) $E_{h}{ }^{\prime}$ is a refinement of $E$, (2) there is an integer $w$ greater than $j+2$ such that each element of $G_{w}{ }^{*}$ that lies in $h^{*}$ lies in a link of $E_{h}^{\prime},(3)$ the end-links of $E_{h}^{\prime}$ contain the end-links of $h$, and (4) there is a proper subchain $D_{h}^{\prime}$ of $D^{\prime}$ such that $D_{h}^{\prime}$ and $E_{h}^{\prime}$ are similar with respect to $D$ and $E$ under a pair of transformations one of which is $A$, and such that if $h$ has a boundary point in common with a link of $G_{j+2}$ that lies in $g_{T}$ (or $g_{U}$ ), then $D_{h}^{\prime}$ contains $d_{1}^{\prime}$ (or $d_{n}^{\prime}$ ).

If $d^{\prime}$ is a link of $D^{\prime}$, let $A^{\prime}\left(d^{\prime}\right)$ be the sum of all links of chains $E_{K}^{\prime}$ or $E_{h}^{\prime}$ which correspond to $d^{\prime}$ under the appropriate similarity transformations. Let $E^{\prime}$ be $A^{\prime}\left(D^{\prime}\right)$, and let $p$ be the largest integer used as a subscript in the above construction. Then $E^{\prime}$ and $\dot{p}$ satisfy the conclusion of the theorem.

CoRollary. Hypothesis: Let $D$ be a chain from $R$ to $S$, and let $D^{\prime}$ be a chain from $R$ to $S$ which is a refinement of $D$, such that if $d^{\prime}$ and $d^{\prime \prime}$ are links of $D^{\prime}$ which lie in the same link of $D$, then $d^{\prime}$ and $d^{\prime \prime}$ are not adjacent. Let $N$ be a subcontinuum of a pseudo-arc $M$, and for each $i$ let $C_{i}^{\prime}$ be the set of all links of $C_{i}$ that contain a point of $N$. Let $T$ and $U$ be points of $N$ which belong to each set 
$C_{i}^{\prime *}$. Let $E$ be a chain from $T$ to $U$ which is a consolidation of a certain $C_{j}^{\prime}$. Let $A$ be a reversible transformation preserving adjacence, such that $A(D)$ is $E$.

Conclusion: There is a chain $E^{\prime}$ from $T$ to $U$, a transformation $A^{\prime}$, and an integer $p$, such that (1) $E^{\prime}$ is a proper consolidation of $C_{p}^{\prime}$ with respect to $E$, and (2) $D^{\prime}$ and $E^{\prime}$ are similar with respect to $D$ and $E$ under the transformations $A$ and $A^{\prime}$.

Proof. For each $i$, let $G_{i}$ be the set whose only element is $C_{i}^{\prime}$. We may choose $j$ large enough so that the hypothesis of Theorem 9 will be satisfied. Let $F$ and $p$ be the chain and the integer described in the conclusion of Theorem 9. Let $E^{\prime}$ be the set of all interiors of sets $f \cdot \mathbb{S}\left(C_{i}^{\prime *}\right)$, where $f$ is a link of $F$. Since $N$ is a continuum, $E^{\prime}$ is a chain.

THEOREM 10. Let $D$ and $E$ be chains, and let $D^{\prime}$ be the amalgam of $E$ with respect to $D$. Let $d^{\prime}$ and $d^{\prime \prime}$ be links of $D^{\prime}$ which lie in the same link of $D$. Then $d^{\prime}$ and $d^{\prime \prime}$ are not adjacent.

THEOREM 11. Let $N$ be a subcontinuum of a pseudo-arc $M$, and let $T$ and $U$ be points of $N$. Let $C$ be a chain from $T$ to $U$ which is a consolidation of $a$ certain $C_{i}^{\prime}$. Let $c$ be a link of $C$, and let $n$ be a natural number. Then there is a chain $D$ from $T$ to $U$, such that (1) $D$ is a proper consolidation of a certain $C_{j}^{\prime}$ with respect to $C$, (2) c contains $n$ links of $D$, and (3) if $c^{\prime}$ is a link of $C$ other than $c$, then $c^{\prime}$ contains only one link of $D$.

Proof. (1) Suppose that $c$ is not an end-link of $C$. Let $b$ and $b^{\prime}$ be the links of $C$ that are adjacent to $c$. Let $X$ be the set of all links of $C_{i+1}^{\prime}$ that have a boundary point in common with $b$. Then no element of $X$ has a boundary point in common with $b^{\prime}$. (See Definition 7, condition (4).) If $n$ is 2 , let the interior of $\mathfrak{s}\left(X^{*}\right)$ and the interior of $\mathbb{S}(c)-\mathfrak{S}\left(X^{*}\right)$ be links of $D$. Let the other links of $D$ be the interiors of the sets $c^{\prime} \cdot \mathbb{S}\left(C_{i+1}^{\prime *}\right)$, where $c^{\prime}$ is a link of $C$ other than $c$. This process can clearly be repeated any desired number of times.

(2) If $c$ is an end-link of $C$, suppose, without loss of generality, that $T$ belongs to $c$; and let $j$ be such that no closure of a link of $C_{j}^{\prime}$ contains $T$ and a point not in $c$. Let $X$ be the set of all links of $C_{j}^{\prime}$ that lie in $c$ and have a boundary point in common with the link of $C$ that is adjacent to $c$, and proceed as before. If the process needs to be repeated, we can split up a nonend-link of the new chain.

THEOREM 12. Let $M$ be a pseudo-arc, let $N$ be a subcontinuum of $M$, and let the sequence $C_{1}, C_{2}, \cdots$ of chains from $P$ to $Q$ be as in Definition 10. Then there are points $R$ and $S$ of $N$, and sequences $X_{1}, X_{2}, \cdots$ and $X_{1}^{\prime}, X_{2}^{\prime}, \cdots$ of chains, and a sequence $T_{1}, T_{2}, \cdots$ of reversible transformations, such that (1) for each $i, X_{i}$ is a chain from $P$ to $Q$, and $X_{i}^{\prime}$ is a chain from $R$ to $S$, (2) for each $i, \mathfrak{S}\left(X_{i}^{*}\right)$ is $M$, and the common part of the sets $\mathfrak{}\left(X_{i}^{\prime *}\right)$ is $N,(3)$ there is a se- 
quence $f_{1}, f_{2}, \cdots$ of positive numbers which converges to 0 , such that each element of $X_{i}+X_{i}^{\prime}$ has diameter less than $f_{i}$, and (4) for each $i, X_{i+1}$ and $X_{i+1}^{\prime}$ are similar with respect to $X_{i}$ and $X_{i}^{\prime}$ under the transformations $T_{i}$ and $T_{i+1}$.

Proof. Let the points $R$ and $S$ of $N$ and the sequence $D_{1}, D_{2}, \cdots$ be as in the conclusion of Theorem 8 . Let $D_{j}$ be the first term of the $D$-sequence which consists of more than five links. Let $D_{j}$ consist of $k$ links. Let $X_{1}^{\prime}$ be $D_{j}$. Let $X_{1}$ be a chain from $P$ to $Q$ which is derived from $C_{1}$ by decomposing a link of $C_{1}$ into enough links so that $X_{1}$ will consist of $k$ links, such that $X_{1}$ is a proper consolidation of a certain $C_{i}$ with respect to $C_{1}$. Let $T_{1}$ be a reversible transformation preserving adjacence, such that $T_{1}\left(X_{1}\right)$ is $X_{1}^{\prime}$. Let $X_{2}$ be the second term of the $C$-sequence which is a refinement of $X_{1}$, and let $Y_{2}$ be the amalgam of $X_{2}$ with respect to $X_{1}$.

For each $i$, let $C_{i}^{\prime}$ be the set of all links of $C_{i}$ that intersect $N$. The hypothesis of the corollary to Theorem 9 is satisfied by $P, Q, R, S, X_{1}, Y_{2}, X_{1}^{\prime}$, and the sequence whose terms are the sets whose only elements are the chains $C_{\boldsymbol{i}}^{\prime}$ for which $i$ is greater than $j$. There is therefore a chain $Y_{2}^{\prime}$ from $R$ to $S$, and a reversible transformation $T_{2}^{\prime}$, such that (1) $Y_{2}^{\prime}$ is a proper consolidation of a certain $C_{p}^{\prime}$ with respect to $X_{1}^{\prime}$, and (2) $Y_{2}$ and $Y_{2}^{\prime}$ are similar with respect to $X_{1}$ and $X_{1}^{\prime}$ under the transformations $T_{1}$ and $T_{2}^{\prime}$. By Theorem 11, there is a chain $X_{2}^{\prime}$ from $R$ to $S$ which is a proper consolidation of a certain $C_{q}^{\prime}$ with respect to $X_{1}^{\prime}$, and a reversible transformation $T_{2}$, such that $X_{2}$ and $X_{2}^{\prime}$ are similar with respect to $X_{1}$ and $X_{1}^{\prime}$ under the transformations $T_{1}$ and $T_{2}$.

Suppose that we have given the first $n-1$ terms of the $X$-sequence, the $X^{\prime}$-sequence, and the $T$-sequence. If $n-1$ is odd, let $X_{n}$ be the second term of the $C$-sequence which is a refinement of $X_{n-1}$, and let $Y_{n}$ be the amalgam of $X_{n}$ with respect to $X_{n-1}$. Proceed as before to obtain the chain $X_{n}$ and the transformation $T_{n}$.

If $n-1$ is even, let $X_{n}^{\prime}$ be the second term of the $D$-sequence which is a refinement of $X_{n-1}^{\prime}$, and let $Y_{n}^{\prime}$ be the amalgam of $X_{n}^{\prime}$ with respect to $X_{n-1}^{\prime}$. Proceed as before to obtain the chain $X_{n}$ and the transformation $T_{n}$.

To verify condition (3), we note that $X_{2 i-1}$ is a refinement of $C_{i}$, and that $X_{2 t}^{\prime}$ is a refinement of $D_{i}$.

TheOREM 13. If $M$ is a pseudo-arc, and $N$ is a subcontinuum of $M$, then $M$ and $N$ are topologically equivalent.

Proof. For each $i$, let the $G_{i}$ of Theorem 2 be the $X_{i}$ of Theorem 12; and let the $H_{i}$ of Theorem 2 be the set of all interiors with respect to $N$ of sets $\sqrt{5}\left(x^{\prime}\right) \cdot N$, where $x^{\prime}$ is a link of the chain $X_{i}^{\prime}$ of Theorem $12, N$ being regarded as space.

UNIVERSITY OF TEXAS Austin Tex. 